\title{
Designing an Informal Learning Curriculum to Develop 3D Modeling Knowledge and Improve Spatial Thinking Skills
}

\author{
Srinjita Bhaduri \\ Tamara Sumner \\ Institute of Cognitive Science \\ Department of Computer Science \\ University of Colorado Boulder \\ Boulder, CO, USA \\ srinjita.bhaduri@colorado.edu \\ sumner@colorado.edu
}

\author{
Katie Van Horne \\ Institute of Cognitive Science \\ School of Education \\ University of Colorado Boulder \\ Boulder, CO, USA \\ Katie.VanHorne@colorado.edu
}

\section{ABSTRACT}

We report on the design and implementation of a 3-week long summer academy introducing high school students to 3D modeling and 3D printing experiences. Supporting youth in developing 3D modeling knowledge can enhance their capacity to effectively use an array of emerging technologies such as Virtual Reality, Augmented Reality, and digital fabrication. We used tools and practices from both formal and informal education, such as storylining, to inform the design of the curriculum. We collected data through surveys, artifacts, observations, screen recordings, and group videos Our findings suggest that (1) emphasizing curricular coherence as a design goal and (2) providing youth with multiple avenues for engaging in 3D modeling can help to: spark youth interest in 3D

Permission to make digital or hard copies of part or all of this work for personal or classroom use is granted without fee provided that copies are not made or distributed for profit or commercial advantage and that copies bear this notice and the full citation on the first page. Copyrights for third-party components of this work must be honored. For all other uses, contact the owner/author(s).

CHI'19 Extended Abstracts, May 4-9, 2019, Glasgow, Scotland UK

2019 Copyright held by the owner/author(s).

ACM ISBN 978-1-4503-5971-9/19/05.

https://doi.org/10.1145/3290607.3299039 


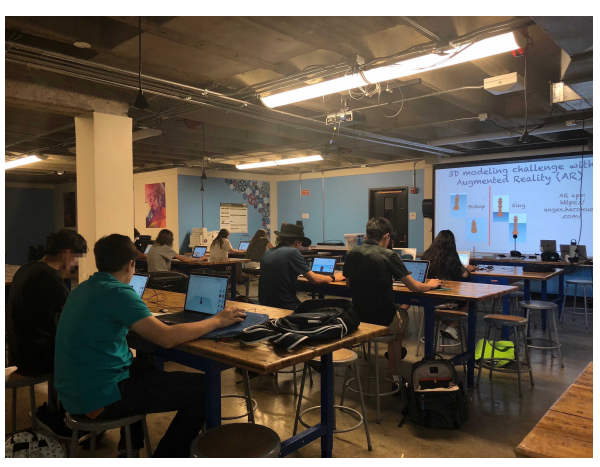

Figure 1: The makerspace where the summer camp took place, youth are working on 3D modeling challenges. printing/modeling, maintain youth engagement in learning activities over the course of several weeks, and provide youth with opportunities to develop their spatial thinking skills.

\section{CCS CONCEPTS}

- Human-centered computing $\rightarrow$ Field studies; User studies; HCl theory, concepts and models;

\section{KEYWORDS}

Youth; 3D Printing; 3D Modeling; Augmented Reality; Spatial Thinking; Storylines; Informal learning; Maker Education

\section{ACM Reference Format:}

Srinjita Bhaduri, Tamara Sumner and Katie Van Horne. 2019. Designing an Informal Learning Curriculum to Develop 3D Modeling Knowledge and Improve Spatial Thinking Skills. In CHI Conference on Human Factors in Computing Systems Extended Abstracts (CHI'19 Extended Abstracts), May 4-9, 2019, Glasgow, Scotland UK. ACM, New York, NY, USA, 8 pages. https://doi.org/10.1145/3290607.3299039

\section{INTRODUCTION}

This article describes a new approach for creating technology-rich, informal learning experiences for adolescent youth that build on and deepen their knowledge and interests in emerging technologies such as 3D modeling, 3D printing, and Unmanned Aerial Vehicles (UAVs). This approach, called "storylining" [9] has emerged from the research base on designing science, technology, engineering, and math (STEM) curriculum to support the vision of learning outlined in the Framework for K-12 Science Education [6]. This vision emphasizes developing learning experiences that are coherent from the students' perspective. That is, during any given learning activity, students should understand how their activities are helping them to solve an overarching engineering design challenge or make progress on a scientific investigation. Researchers have proposed storylines as a promising approach for developing curriculum that is coherent [9].

In this project, we adapted the storyline technique to develop a summer camp that provided high school youth with opportunities to gain in-depth experiences designing and creating with emerging technologies found in makerspaces. We report on the storyline design of the curriculum, our experiences implementing it with 12 youth over 3-weeks, the strengths and weaknesses of it, and the utility of the storylining tool for similar educational settings. Although there is growing body of research on the impact of makerspaces and maker technologies on youth, there are few published studies discussing how to design curriculum to provide youth with in-depth experiences using these technologies (Figure 1). This research addresses this gap. 
Three questions guided the design of our curriculum:

(1) To what degree and in what ways can $3 \mathrm{D}$ modeling and printing be made approachable to youth with different skillset?

(2) What other technologies can be used to support youth understanding of the strengths and weaknesses of 3D models?

(3) How can learning 3D modeling skills help enhance youth spatial thinking skills?

\section{Sidebar 1: Research Questions}

\section{DESIGN: THE CURRICULUM STORYLINE}

A storyline is a way to represent a sequence of lessons where each lesson is motivated by youth questions about the design challenge they are solving [9]. During each lesson, youth use science and engineering practices (SEPs) [6]- such as asking questions and defining problems, planning and carrying out investigations, or designing and testing solutions, in turn, supports youth in generating more questions, which motivate the next activity in their learning sequence. Thus, when participating in learning environments based on storylines, students are co-constructing their learning experiences by articulating the questions they are working on. In this project, we adapted the storylining technique to support the design of a 3-week long informal learning summer camp. Our goal was to provide students with a coherent learning experience incorporating an array of advanced technologies that are found in makerspaces, including 3D printing and 3D modeling (see Sidebar 1 for research questions).

The storyline underpinning our curriculum design is shown in Figure 2. Youth were asked to develop a solution to a design challenge: how to mimic a town that has been damaged and isolated due to a natural disaster and use tools to survey and provide relief to the town. This design challenge was adapted from a longer 16-week curriculum (Engineering Experiences) [2]. We modified this preexisting curriculum to fit our goal of making 3D modeling approachable to youth and included several opportunities for 3D modeling and printing throughout the camp. The curriculum was designed considering the driving questions youth would need to answer to solve the problem [9]. In Figure 2, the driving questions (color coded in pink) for each of the activities are aligned with tools/technologies (color coded in green) youth had to learn and use to be able to address each driving question. Figure 2 also shows the SEPs that youth would need to engage in to make progress on the anticipated driving questions, such as defining problems and planning and carrying out investigations. The goal was for youth to be immersed in using these practices to figure out how to design, test, and iterate on their designs of a partially 3D printed UAV obstacle course.

In addition to providing youth with opportunities to learn 3D modeling and printing for obstacle course we wanted to study the degree to which using 3D modeling tools over time can enhance youth spatial thinking skills, a linkage identified through prior research [10]. However, prior research has also reported a number of usability and learnability problems when using 3D modeling softwares $[1,5]$. Learning 3D modeling can be daunting and requires a serious time commitment [7]. Some 3D modeling tools limit students as their design skills grow and other tools may be difficult to learn initially, resulting in frustration and disengagement of students [8]. Since we could not anticipate the prior experiences of the youth participating in the program, we designed the curriculum to include 4 different approaches to 3D modeling to support youth with varying skill-sets: (1) introduce youth to a variety of 3D CAD softwares and enable them to choose ones that are appropriate to their skill level (see Figure 2, list of tools used), (2) illustrate the various approaches for creating a 3D model 


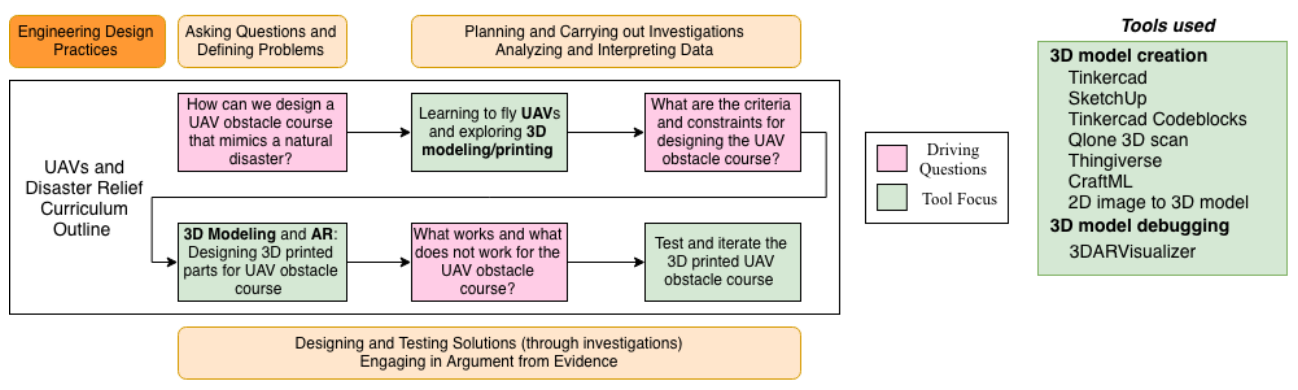

Figure 2: Flow and Driving Questions of the curriculum.

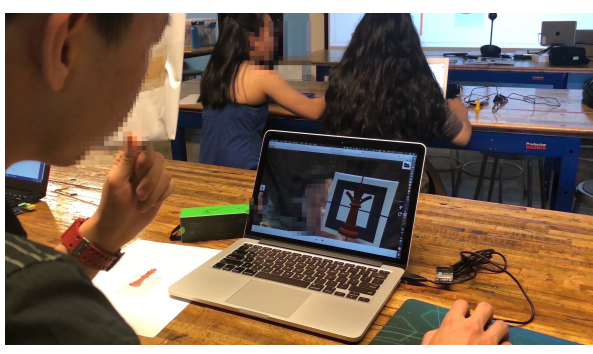

Figure 3: Youth testing 3D model using the 3DARVisualizer app with an AR marker image. such as scanning 3D objects, converting 2D images to 3D models, (3) support novice modelers in debugging their 3D models prior to printing: we created an augmented reality tool - 3DARVisualizerthat enables youth to visualize and rotate 3D models in order to detect gaps or alignment issues that would cause a print to fail, and (4) support design-by modification: downloading preexisting 3D models and modifying them.

\section{IMPLEMENTATION: CREATIVE TECHNOLOGIES ACADEMY}

Our study was part of a 3-week long summer camp called Creative Technologies Academy. The curriculum for the camp was designed and implemented by an interdisciplinary partnership that included scientists from University Corporation for Atmospheric Research (UCAR), and learning scientists and computer scientists from the University of Colorado Boulder. Our partners have expertise in 3D modeling, 3D printing, human-computer interaction $(\mathrm{HCl})$, cognitive science, education, curriculum design, and atmospheric sciences. The Academy consisted of 12 high-school students (5 female) between 14 to 17 years of age; 4 students were visiting from outside the United States to participate in the program. The participants had varied experience in $3 \mathrm{D}$ modeling, ranging from absolute newcomers to knowledgeable users. The camp took place in a makerspace which included 3D printers, a UAV flying space, and computers (Figure 1) and was facilitated by the first author. The camp met daily from Monday-Friday for 3 weeks; each day included 4 hours of instructional time and 1.5 hours of break. At the beginning of each session youth were shown motivational and informational videos related to fabrication technologies to generate discussion topics. Youth worked in groups to address the design challenge and individually to explore the different tools. 


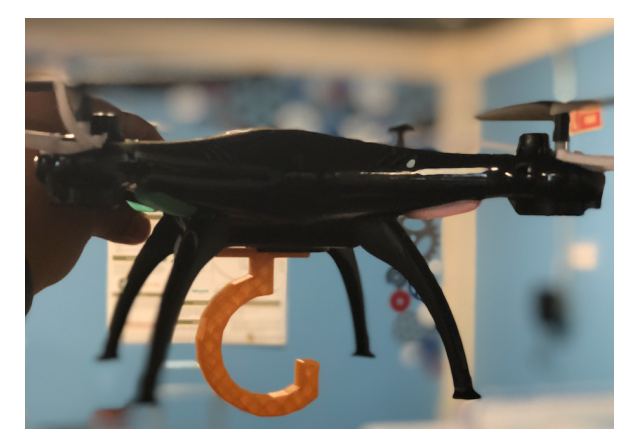

Figure 4: Hook like attachments for UAVs to carry supplies.

\section{Week 1: How can we design a UAV obstacle course that mimics a natural disaster?}

To answer this question, youth were introduced to the importance of UAVs in disasters. Then they learned 3D modeling and printing, starting with a short video and demonstration of the process of additive manufacturing. Youth formed groups of 2-4 members to work on UAV obstacle courses. To plan and design the partially 3D printed UAV obstacle course, they began using a beginner-friendly 3D modeling tool Tinkercad. In order to provide youth with in-depth 3D modeling experience they were given individual 3D modeling tasks. Participants were given sample images of 3D chess pieces to design, personalize, and 3D print these pieces. In following sessions, youth were introduced to AR and the AR based 3D model debugger tool that our team developed: 3DARVisualizer [3]. Youth could upload .stl files of their 3D models and use marker images or visual-cues to augment 3D models on the images using 3DARVisualizer, with options to rotate, scale, and test 3D models for gaps, alignment, and overall printability (Figure 3). This time, youth were given sample images of different 3D chess pieces to design and personalize. They used the 3DARVisualizer to test their models and check for overall printability. Another 3D modeling approach they learned was Tinkercad Codeblocks. This 3D modeling tool uses blocks of code to create 3D structures. Youth also learned how to convert 2D images to 3D models and personalize them. These different techniques to 3D modeling provided participants with ideas to design the UAV obstacle course. They learned to fly UAVs and conducted UAV performance test like battery-life, weight test. It helped them think about the driving question around the different criteria and constraints for designing the obstacle course.

\section{Week 2: What are the criteria and constraints for designing the UAV obstacle course?}

In this week, youth continued their work on the obstacle course and brainstormed multiple ideas for criteria and constraints for it. Then they narrowed down to the ones that were most feasible considering the time, space, and resources at hand. They designed and 3D printed objects like payloads, hook-like attachments for the UAVs (Figure 4), and other 3D objects for their obstacle course like connectors and 3D printed UAV launch pad. Having learned different approaches to 3D modeling, in this week we gave youth the freedom to choose any 3D modeling tool they preferred. They explored ways to scan 3D objects and modify the 3D models created from the scans. We took participants to an outdoor space to make sketches of object(s) around them. We also taught them another approach to 3D modeling using CraftML [11], which uses HTML and CSS tags to create 3D models. By the end of this week, each group designed prototypes and 3D printed objects for their obstacle courses.

\section{Week 3: What works and what does not work for the UAV obstacle course?}

In the final week, youth worked on testing their obstacle courses. We allowed the participants to make their own 3D models or download them from Thingiverse and 3D print them. This was to support their tinkering and making abilities and provide opportunity to explore this field. On the second to 


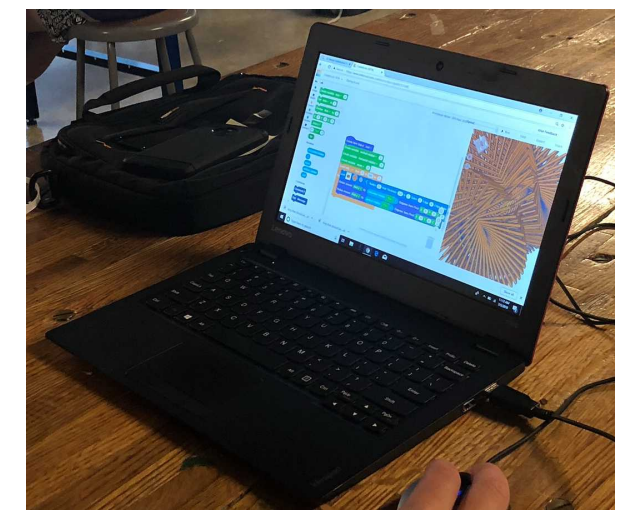

Figure 5: Youth using Tinkercad Codeblocks to design complex 3D models.

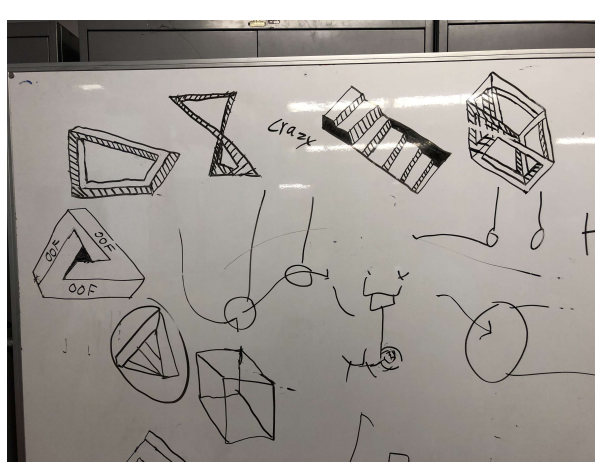

Figure 6: Spatially complex sketches made by youth. last session, the groups tested their obstacle courses and each group was able to try the other group's obstacle course. On the final day, youth presented their projects at an expo where students from different camps were presenting their work.

\section{FINDINGS}

Through data collected from surveys, youth artifacts, observations, screen recordings, and youth team videos we present our findings around our research questions.

\section{Making 3D modeling and printing approachable to youth with different skill-sets}

Our observations suggest that making 3D modeling and printing available to youth in different forms helps spark their interest in learning and exploring these tools. For example, by introducing Tinkercad Codeblocks youth who preferred programming (as opposed to designing with primitives) were highly motivated to design complex shapes and structures using it (Figure 5). The surveys support this finding, like one participant noted "the best thing was learning so many new techniques to 3d printing" [P3]. We also observed that youth learning 3D modeling for the first time preferred to use Tinkercad, while youth with more experience preferred SketchUp or other complex tools like Maya or Blender Youth were also transitioning to more advanced tools during the 3-week period as they grew more comfortable visualizing, rotating, and analyzing 3D models for printability.

\section{Supporting youth understanding of the strengths and weaknesses of 3D models}

Previous work suggests that failed 3D prints are often discouraging to students [4]. To address this issue, we developed $3 D A R$ Visualizer and youth used it to test their $3 \mathrm{D}$ models. Testing the models before printing them resulted in successful prints and youth were able to test for any gaps, alignment issues, and overall printability of the models. In the surveys, 7 of the 12 youth rated $3 D A R$ Visualizer to be somewhat useful or very useful in debugging their 3D models. One youth with less 3D modeling experience said, "I was able to see many open spaces in my chess pieces in particular" [P6]. While others who had prior experience with 3D modeling did not find it very helpful (5 of the 12). However, several of them suggested extensions to the debugger to make it more useful, such as allowing them to check the size, shape, and fit of the model using the app. For example, one participant said, "It only works on a marker which isn't scaled properly, so this AR isn't as useful for checking sizes" [P4].

\section{Enhancing spatial thinking skills in youth}

The surveys suggested that youth had a better understanding of the 3D space after working with a variety of 3D modeling tools. For instance, a participant said, "Figuring out how to center things has been harder but in the last couple of days it has gotten easier" [P9]. Data collected from pre- and post-spatial thinking tests (tests adapted from Spatial Intelligence and Learning Center) suggests 


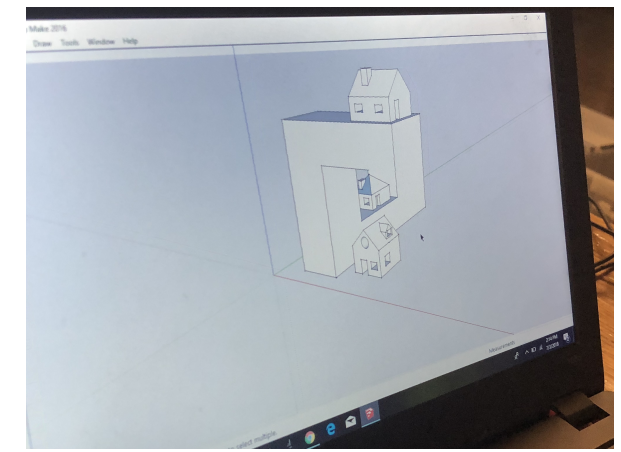

Figure 7: 3D model of a visually complex house designed by a youth using SketchUp.

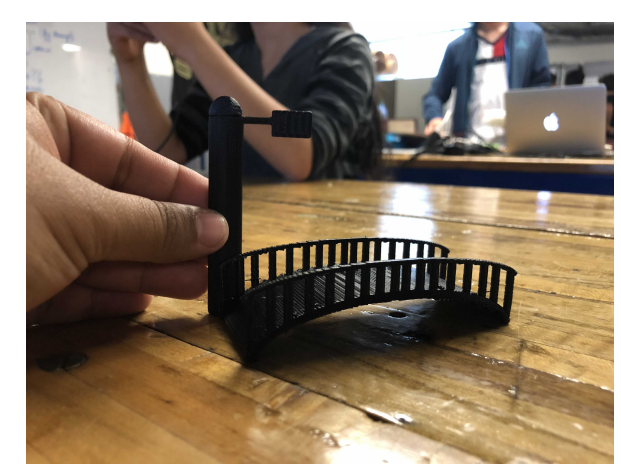

Figure 8: 3D print of a bridge and a lamp post that a participating youth created as part of the outdoor sketching activity. that there was a change in spatial thinking skills for 5 of the 12 participants. These participants also showed improvements when playing a spatial reasoning game. While 7 of the 12 participants showed no improvement on the pre- and post-tests, our observations suggest that they were deeply engaged in spatial thinking tasks: we saw youth making spatially complex sketches (see Figure 6). And, participants played spatial games, competing to create the most complex 3D objects (see Figure 7).

\section{DISCUSSION}

We identified some advantages and disadvantages of the design and implementation of our curriculum

\section{Curriculum Design and Coherence}

From our findings we would like to recommend using storylining to design a coherent curriculum since it provides youth with hands-on opportunities to work on real-world problems. Due to the fact that 3D modeling and printing are time-intensive and can easily lead to disengagement of youth, it is important to design an environment that supports creativity and robust learning while not sacrificing the engagement level. For example, we used several forms of 3D modeling, helping youth with prior experience in 3D modeling to explore other techniques. The outdoor sketching activity helped youth to explore the world outside their classroom and was a great experience for them to actually design and print something they saw (see Figure 8). Even though 3D printing is "fun" and a relatively new and innovative technology in educational or maker spaces, 3D printing alone is not always enough to keep the youth engaged [1]. There should be a minimum level of scaffolding in the lesson plans, tasks, and projects. While some participants struggled with the 3D modeling tasks others were interested in tangible outcomes like their 3D prints. We found that having different levels for each tasks and giving youth their own tinkering time worked for promoting creativity. Moreover, we noticed that providing too much time on group projects might lead to youth disengagement from their projects. So we need to rethink our driving questions to include more time for individual activities. Thus, we suggest a need to structure maker curriculum in a way that there is good balance between the concepts and tools being taught versus the time spent working on them.

\section{Choosing wide range of softwares and tools to spark the interest}

When selecting 3D modeling softwares educators and instructors should keep in mind the personal experiences, expertise, and abilities of the youth with these technologies. This helps reduce the level of frustration that novice users have when dealing with difficult terminologies and user-interfaces. Using different approaches to 3D modeling and allowing youth to choose from these worked great for teaching a group of youth with varied abilities and experiences. Additionally, using 3D model debugging tools like 3DARVisualizer can lower the frustration level from failed prints due to the opportunities it provides to navigate models in 3D space and motivate youth to understand the rapid prototyping opportunities that fabrication technologies offer. 


\section{CONCLUSION AND WHAT'S NEXT}

Our case study suggests that designing a coherent curriculum using storylining technique to teach 3D modeling and printing can be valuable for promoting and maintaining interest in these technologies and in the process enhance spatial awareness in youth. While there is work that needs to be done to generalize our findings, the current results are promising, potentially offering a new way for educators to design coherent curriculum for makerspaces. Moving forward, we plan to make the curriculum modular so that it can be incorporated in different informal settings. For example, to solve a locally relevant problem the storyline can be modified to fit the purpose of a particular culture and in turn youth can have rich experience learning the tools to solve problems. Moreover, we would like to modify 3DARVisualizer and incorporate some of the changes that youth suggested like easier navigation and real-life size comparison of 3D models. Finally, our plan is to conduct workshops and interviews with makerspace instructors or teachers and find ways to make the AR app useful for students.

\section{REFERENCES}

[1] Alexander Berman, Elizabeth Deuermeyer, Beth Nam, Sharon Lynn Chu, and Francis Quek. 2018. Exploring the 3D printing process for young children in curriculum-aligned making in the classroom. In Proceedings of the 17th ACM Conference on Interaction Design and Children. ACM, 681-686.

[2] Srinjita Bhaduri, Katie Van Horne, John Daniel Ristvey Jr., Randy Russell, and Tamara Sumner. 2018. From Toys to Tools: UAVs in Middle-school Engineering Education (RTP). In 2018 ASEE Annual Conference \& Exposition. ASEE Conferences, Salt Lake City, Utah. https://peer.asee.org/30546

[3] Srinjita Bhaduri, Katie Van Horne, Peter Gyory, Hannie Ngo, and Tamara Sumner. 2018. Enhancing 3D Modeling with Augmented Reality in an After-school Engineering Program (Work in Progress). In 2018 ASEE Annual Conference \& Exposition. ASEE Conferences, Salt Lake City, Utah. https://peer.asee.org/30424

[4] Erin Buehler, William Easley, Samantha McDonald, Niara Comrie, and Amy Hurst. 2015. Inclusion and education: 3D printing for integrated classrooms. In Proceedings of the 17th International ACM SIGACCESS Conference on Computers \& Accessibility. ACM, 281-290.

[5] Parmit Chilana, Nathaniel Hudson, Srinjita Bhaduri, Prashant Shashikumar, and Shaun Kane. 2018. Supporting Remote Real-time Expert Help: Opportunities and Challenges for Novice 3D Modelers. In Proceedings of the IEEE Symposium on Visual Languages and Human-Centric Computing (VL/HCC'18). IEEE.

[6] National Research Council et al. 2012. A framework for K-12 science education: Practices, crosscutting concepts, and core ideas. National Academies Press.

[7] James Floyd Kelly. 2014. 3D Modeling and Printing with Tinkercad: Create and Print Your Own 3D Models. Que Publishing.

[8] Ben Lafreniere and Tovi Grossman. 2018. Blocks-to-CAD: A Cross-Application Bridge from Minecraft to 3D Modeling. In The 31st Annual ACM Symposium on User Interface Software and Technology. ACM, 637-648.

[9] Brian J Reiser. 2014. Designing coherent storylines aligned with NGSS for the K-12 classroom. In National Science Education Leadership Association Meeting. Boston, MA.

[10] Andrej Šafhalter, Karin Bakracevic Vukman, and Srečko Glodež. 2016. The effect of 3D-modeling training on students' spatial reasoning relative to gender and grade. Journal of Educational Computing Research 54, 3 (2016), 395-406.

[11] Tom Yeh and Jeeeun Kim. 2018. CraftML: 3D Modeling is Web Programming. In Proceedings of the 2018 CHI Conference on Human Factors in Computing Systems. ACM, 527. 